\title{
CHARACTERIZATION OF FINITE DEDEKIND GROUPS
}

\author{
REINHOLD BAER \\ To the memory of TADAsI NaKayama
}

If $U=U(0), U(i)$ is a maximal subgroup of $U(i+1)$ and $U(n)=V$, then we term the $U(i)$ a densest chain connecting $U$ and $V$; and $n$ is the length of this chain. The subgroup $U$ of $V$ will furthermore be termed an $n$-uniserial subgroup of $V$ if there exists one and only one densest chain connecting $U$ and $V$ and if its length is $n$. The principal aim of this note is to give characterizations of dedekind groups [ = groups all of whose subgroups are normal] in terms of the normality of uniserial subgroups. We quote one of our results:

The finite group $G$ is a dedekind group if, and only if, all uniserial subgroups of $G$ are normal and normal subgroups of $G$ normalize their 2 -uniserial subgroups [Corollary 2.14].

Our principal results [Theorems 2.10 and 3.3] are a little more differentiated. 'The method employed consists in an exact determination of all finite groups that are not dedekind groups, though all their proper epimorphic images are dedekind groups and which in addition meet certain definite requirements [Proposition 2.1].

\section{Notations}

$\mathbf{z} G=$ center of $G$.

$\mathbf{c} U=\mathbf{c}_{\theta} U=$ centralizer of $U$ in $G$.

$\boldsymbol{x} \circ \boldsymbol{y}=\boldsymbol{x}^{-1} y^{-1} x y$

$x^{y}=x(x \circ y)=y^{-1} x y$

$U \circ V=$ subgroup generated by all the $u \circ v$ with $u$ in $U$ and $v$ in $V$.

$G^{\prime}=G \circ G=$ commutator subgroup of $G$.

Factor of $G=$ epimorphic image of subgroup of $G$.

$\{\cdots\}=$ subgroup generated by the enclosed set.

All groups considered are finite!

Received January 20, 1965. 


\section{Uniseriality}

If $U$ is a subgroup of $G$, and if the $U(i)$ are subgroups of $G$ such that

$U=U(0), U(i)$ is a maximal subgroup of $U(i+1), U(n)=G$,

then we term the $U(i)$ a densest series connecting $U$ and $G$; and $n$ is the length of this series. The subgroup $U$ of $G$ is termed uniserial, if there exists (one and) only one densest series connecting $U$ and $G$; and the uniserial subgroup $U$ of $G$ is termed $n$-uniserial, if $n$ is the length of the uniquely determined densest series connecting $U$ and $G$.

Clearly the subgroup $U$ of $G$ is uniserial if, and only if, the set of subgroups of $G$, containing $U$, is [linearly] ordered by inclusion; and $U$ is $n$ uniserial, if $n+1$ is the number of subgroups of $G$ which contain $U$.

It is clear that $G$ is its one and only one 0-uniserial subgroup and that a subgroup $M$ of $G$ is maximal if, and only if, it is 1-uniserial. One verifies furthermore without any difficulty that 1 is an $n$-uniserial subgroup of $G$ if, and only if, $G$ is cyclic of order $p^{n}$ (for some prime $p$ ).

(1.1) The following properties of the subgroup $U$ of the group $G$ and the positive integer $n$ are equivalent:

( $i$ ) $U$ is an n-uniserial subgroup of the group $G$.

(ii) There exists one and only one maximal subgroup $M$ of $G$ which contains $U$; and $U$ is an $(n-1)$-uniserial subgroup of $M$.

(iii) There exists one and only one subgroup $B$ of $G$ of which $U$ is a maximal subgroup; and $B$ is an $(n-1)$-uniserial subgroup of $G$.

The simple proof of this criterion may be left to the reader. The interest of this criterion stems from the fact that it contains two inductive definitions of uniseriality.

If $U$ is an $n$-uniserial subgroup of $G$, and if $B$ is a subgroup of $G$ which contains $U$, then $B$ is an $i$-uniserial subgroup of $G$ and $U$ is an $(n-i)$-uniserial subgroup of $B$. The converse of this statement is false, as may be seen from the example of the elementary abelian groups of order $p^{2}$.

Lemma 1.2. Suppose that $\sigma$ is an epimorphism of $G$ upon $H$.

(a) If $U$ is an n-uniserial subgroup of $G$, then $U^{\sigma}$ is an i-uniserial subgroup of $H$ with $i \leq n$. 
(b) If $V$ is an $n$-uniserial subgroup of $H$, then the inverse image $V^{0^{-1}}$ is an $n$-uniserial subgroup of $G$.

Proof. Suppose that $U$ is an $n$-uniserial subgroup of $G$ and denote by $U(i)$ with $0 \leq i \leq n$ the uniquely determined densest series connecting $U$ and $G$. If $B$ is a subgroup of $H$ containing $U^{\sigma}$, then $U \subseteq B^{\sigma^{-1}}$ so that $B^{\gamma^{-1}}=U(i)$ for some suitably selected $i$. If $C$ is another subgroup of $H$ containing $U^{\sigma}$, then $0^{\gamma^{-1}}=U(j)$ for some $j$; and it is clear that $B \subseteq C$ if, and only if, $i \leq j$. Consequentylthe subgroups of $H$ containing $U^{\sigma}$ form a densest series connecting $U^{\sigma}$ and $H$; this densest series is the only densest series connecting $U^{\circ}$ and $H$; and its length does not exceed $n$. This proves (a).

If $K$ is the kernel of $\sigma$, then $\sigma$ induces an isomorphism of $G / K$ upon $H$ and consequently an isomorphism of the lattice of subgroups of $G$ containing $K$ upon the lattice of subgroups of $H$. All the inverse images of subgroups of $H$ contain $K$; and (b) is an immediate consequence of these remarks.

The group $G$ is said to be of exponent $e$ if $G^{e}=1$. Note that every multiple of $e$ is likewise an exponent of $G$. If $e$ is not divisible by the $n$-th power of any prime, then we shall say that $G$ is of $n$-th power free exponent. One verifies easily the equivalence of the following properties of a group $G$ :

(i) $G$ is of $n$-th power free exponent.

(ii) If $P$ is a [cyclic] $p$-subgroup of $G$, then $p^{p^{n-1}}=1$.

(iii) If 1 is an $i$-uniserial subgroup of the subgroup $U$ of $G$, then $i<n$.

\section{The characterization of dedekind groups within the class of nilpotent groups}

A group is termed a dedekind group, if all its subgroups are normal subgroups. These groups are completely classified by the following well known

Structure Theorem. A non-abelian group is a dedekind group if, and only if, it is the direct product of a quaternion group, an elementary abelian 2-group and an abelian group of odd order.

For a proof see, for instance, Hall [p. 190, Theorem 12.5.4]. We begin our discussion by proving a useful reduction theorem.

Proposition 2.1. If the nilpotent group $G$ is not a dedekind group, though all its proper epimorphic images are dedekind groups, then 
( $I$ ) $G$ is a p-group;

( II ) $\mathrm{z} G$ is cyclic;

(III) $G^{\prime}$ is of order $p$;

( $I V) \quad G^{\prime} \cdot G^{p} \subseteq \mathrm{zG}$;

$(V)$ The following properties of the subgroup $S \neq 1$ of $G$ are equivalent:

( a ) $\mathrm{S}$ is normal;

( $b$ ) $S \cap \mathrm{z} G \neq 1$;

( c ) $G^{\prime} \subseteq S$.

(VI) If the order of the cyclic subgroup $C$ of $G$ is divisible by $p^{2}$, then $G^{\prime} \subset C$ and $C$ is normal.

(VII) Non-normal subgroups of $G$ are elementary abelian.

Proof. Assume by way of contradiction the existence of two different normal subgroups $X$ and $Y$ of $G$ each of order a prime - these primes may be equal or different.' Since $G$ is nilpotent, $X Y \subseteq \mathbf{z} G$; see Hall [p. 49, Theorem 4.3.4]. Consider a cyclic subgroup $S$ of $G$. If $S \cap \mathrm{z} G \neq 1$, then $G /(S \cap \mathrm{z} G)$ is by hypothesis a dedekind group so that $S /(S \cap \mathrm{z} G)$ is normal in $G /(S \cap \mathrm{z} G)$ implying the normality of $S$ in $G$. Assume next $S \cap \mathbf{z} G=1$. Since, by hypothesis, $G / X$ and $G / Y$ are dedekind groups, $S X$ and $S Y$ are normal subgroups of $G$. Hence $S X \cap S Y$ is a normal subgroup of $G$. If $x$ is an element in $X \cap S Y$, then $x=s y$ for $s$ in $S$ and $y$ in $Y$; and it follows that $s=x y^{-1}$ belongs to $S \cap X Y \subseteq S \cap \mathrm{z} G=1$. Hence $x=y$ belongs to $X \cap Y=1$, proving that $X \cap S Y=1$. Consequently it follows from Dedekind's modular law that

$$
S=S(X \cap S Y)=S X \cap S Y
$$

is a normal subgroup of $G$. Thus all cyclic subgroups of $G$ are normal so that $G$ is a dedekind group, a contradiction, proving:

(a) There exists at most one normal cyclic subgroup of order a prime.

Every subgroup of $\mathrm{z} G$ is a normal subgroup of $G$. Application of (a) shows that $\mathrm{z} G$ possesses at most one subgroup of order a prime. Applying a well known theorem from the theory of finite abelian groups-see, for instance, Fuchs [p. 80, Corollary 24.4]-it follows that

(b) $\mathrm{z} G$ is a cyclic $p$-group.

As a nilpotent group $G$ is the direct product of its primary components. If $C$ is one of the primary components of $G$, then $\mathrm{z} C=C \cap \mathrm{z} G$; and $C \neq 1 \mathrm{im}$ - 
plies $\mathrm{z} C \neq 1$-see, for instance, Hall [p. 47, Theorem 4.3.1]. Application of (b) shows that only the $p$-component of $G$ is different from 1 ; and thus we see that

(c) $G$ is a $p$-group.

Since $G$ is not a dedekind group, $G$ is a $p$-group, not 1 ; and this implies $\mathbf{z} G \neq 1$. Consequently $\mathbf{z} G$ contains one [and by (b) only one] cyclic subgroup $P$ of order $p$. Assume by way of contradiction that $G / P$ is not abelian. Since $G / P$ is by hypothesis a dedekind group and by (c) a $p$-group, it follows from the Structure Theorem that

$G / P=Q \times E$ is the direct product of a quaternion group $Q$ and an elementary abelian 2-group $E$ so that in particular $p=2$.

It follows in particular that two elements in $G / P$ do not commute if, and only if, they are of order 4 and transform each other into their inverses. Consider an element $P a$ of order 4 in $G / P$. Then $P a^{2}$ is of order 2 so that $a^{2} \neq 1$. If $x$ is any element in $G$, then

$$
a^{x} \equiv a^{ \pm 1} \bmod P
$$

and this implies $\left(a^{2}\right)^{\times}=a^{2 \times}=a^{ \pm 2}$ since $P$ is a subgroup of order 2 of $\mathrm{z} G$. Consequently $\left\{a^{2}\right\}$ is a normal subgroup, not 1 , of $G$; and as a cyclic 2-group, $\left\{a^{2}\right\}$ contains one and only one element of order 2 which is clearly a center element of the 2-group $G$. By (b) there exists at most one element of order 2 in $\mathrm{z} G$; and this is clearly contained in $P$. Since $P$ is cyclic of order 2 , it follows that $P \subseteq\left\{a^{2}\right\} \subseteq\{a\}$. Since $G / P$ is, by hypothesis, a dedekind group, $\{a\} / P$ is a normal subgroup of $G / P$ so that $\{a\}$ is a normal subgroup of $G$. Since $\{P a\}=\{a\} / P$ has order 4 and $P$ has order 2 , the cyclic group $\{a\}$ has order 8.

Denote now by $R$ the uniquely determined subgroup of $G$ which satisfies $P \subseteq R$ and $R / P=Q$. Consider an element $x$ in $R$. If $P x$ is of order 4 , then we have shown just now that $\{x\}$ is a normal subgroup of $G$; and the same is trivially true if $P x=1$. If finally $P x$ has order 2 , then $P x=P y^{2}$ for some $y$ in $R$, since $Q$ is a quaternion group. Since $P y$ is an element of order 4 in $Q$, the result of the preceding paragraph of our proof implies $P \subseteq\{y\}$; and from $\{x\} \subseteq P\{y\}=\{y\}$ we deduce that the characteristic subgroup $\{x\}$ of the normal subgroup $\{y\}$ of $G$ is itself a normal subgroup of $G$. Hence $R$ is a dedekind 2-group, containing elements of order 8 ; and such a group is by the Structure 
Theorem abelian. But $R / P=Q$ is a quaternion group which is not abelian. This is a contradiction, proving that $G / P$ is abelian. Hence $1 \subset G^{\prime} \subseteq P$, as $G$ is not a dedekind group and therefore not abelian. But $P$ is cyclic of order a prime, so that $G^{\prime}=P$. Hence, by (b),

(d) $G^{\prime}$ is the uniquely determined subgroup of order $p$ of $\mathrm{z} G$.

If $x$ and $y$ are elements in $G$, then their commutator $x \circ y$ belongs by (d) to $\mathrm{z} G$ and satisfies $(x \circ y)^{p}=1$. Hence

$$
x^{y}=x(x \circ y), x^{y^{i}}=x(x \circ y)^{i}, x^{y^{p}}=x
$$

so that $x$ and $y^{p}$ commute for every $x$ and $y$ in $G$, proving that $y^{p}$ belongs to $\mathrm{z} G$ and that therefore

(e) $G^{p} \subseteq \mathbf{z} G$.

Consider now a subgroup $S \neq 1$ of $G$. If $S$ is normal, then $S \cap \mathrm{z} G \neq 1$, since $G$ is a $p$-group; see, for instance, Kurosh [II, p. 165]. But $S \cap \mathrm{z} G \neq 1$ implies $G^{\prime} \subseteq S$ by $(\mathrm{d})$; and $G^{\prime} \subseteq S$ naturally implies the normality of $S$. Thus we have shown:

(f) The following three properties of the subgroup $S \neq 1$ of $G$ are equivalent:

$$
G^{\prime} \subseteq S ; S \text { is normal; } S \cap \mathbf{z} G \neq 1 .
$$

If the order of the cyclic subgroup $C$ of $G$ is divisible by $p^{2}$, then $1 \subset C^{p} \subseteq \mathrm{z} G$ by (e) so that $G^{\prime} \subseteq C^{p}$ by (f). Hence

(g) If the order of the cyclic subgroup $C$ of $G$ is divisible by $p^{2}$, then $G^{\prime} \subseteq C$ and $C$ is normal.

Suppose now that the subgroup $S$ of $G$ is not normal. Then $G^{\prime}$ is by (f) not part of $S$ so that $S \cap G^{\prime}=1$ by (d). Hence $S^{\prime}=1$. We deduce furthermore from ( $\mathrm{g}$ ) that $S$ cannot contain cyclic subgroups of order $p^{2}$, proving that

(h) non-normal subgroups are elementary abelian.

Corollary 2.2. The group $G$ is a dedekind group if, and only if, $G$ is nilpotent and $G /\left(G^{\circ} G^{\prime}\right)$ is a dedekind group.

Proof. That dedekind groups are nilpotent, is a consequence of the Structure Theorem; and it is obvious that epimorphic imgages of dedekind groups are dedekind groups.- Assume conversely that $G$ is nilpotent and $G /\left(G^{\circ} G^{\prime}\right)$ is a dedekind group. If $G$ were not a dedekind group, then there would exist an epimorphic image $H$ of $G$ with the following three properties: 
(1) $H$ is not a dedekind group;

(2) every proper epimorphic image of $H$ is a dedekind group;

(3) $H /\left(\dot{H} \circ H^{\prime}\right)$ is a dedekind group.

Because of (1) and (2) we may apply Lemma 2.1, (IV) to prove the commutativity of $H / \mathbf{z} H$. Note next that $Y / X$, for $X$ a normal subgroup of $Y$, is a group with abelian central quotient group if, and only if, $Y^{\circ} Y^{\prime} \subseteq X$. Thus the commutativity of $H / \mathbf{z} H$ implies $H \circ H^{\prime}=1$; and it follows from (3) that $H$ is a dedekind group, contradicting (1), and proving the sufficiency of our conditions.

PROPOSITION 2.3. If the nilpotent group $G$ is not a dedekind group, though all its proper epimorphic images are, and if normal subgroups of $G$ normalize their 2-uniserial subgroups, then $G$ is a p-group and there exists a cyclic normal subgroup $C$ of $G$ and a cyclic subgroup $E$ of order $p$ of $G$ with the following properties :

(a) $G=E C$ and $1=E \cap C$.

(b) $C^{p}=\mathrm{z} G$ and $G^{\prime}$ is the uniquely determined subgroup of order $p$ of $C$.

(c) If $p^{n}$ is the order of $C$, then $2<n$ and $E$ is an $n$-uniserial subgroup of $G$.

Proof. Application of Proposition 2.1 shows that $G$ is a $p$-group with the following properties:

(1) $\mathrm{z} G$ is cyclic ; $G^{\prime}$ is of order $p ; G^{\prime} \cdot G^{p} \subseteq \mathbf{z} G$.

(2) The following three properties of the subgroup $S \neq 1$ of $G$ are equivalent:

$$
G^{\prime} \subseteq S ; S \text { is normal; } S \cap \mathrm{z} G \neq 1 ;
$$

(3) If the order of the cyclic subgroup $C$ of $G$ is divisible by $p^{2}$, then $G^{\prime} \subset C$ and $C$ is normal.

(4) Non-normal subgroups of $G$ are elementary abelian.

Since $G$ is not a dedekind group, there exist non-normal subgroups of $G$; and amongst these there is one $E$ of maximal order. Though this subgroup $E$ is not uniquely determined, we shall retain it throughout the remainder of the proof.

An immediate application of (1)-(4) shows that

(5) $E$ is elementary abelian and $E \cap G^{\prime}=E \cap \mathbf{z} G=1$.

Let $N=\left\{E^{G}\right\}$ be the normal subgroup of $G$, spanned by $E$. Then $E \subset N$, since $E$ is not normal. If $S$ is a subgroup of $G$ with $E \subset S$, then $S$ is a normal 
subgroup of $G$ because of the maximality of $E$. Hence $N \subseteq S$. Thus we see that

(6) $N=\left\{E^{G}\right\}$ is the one and only one subgroup of $G$ of which $E$ is a maximal subgroup.

Since $E$ is a maximal subgroup of $E G^{\prime}$ by (5) and (1), we have by (5) and (6):

(7) $N=E G^{\prime}$ is elementary abelian; and $E \cap G^{\prime}=1$.

Suppose that $X$ is a subgroup of order $p$; and assume by way of contradiction that $X \neq N$. Then $N \cap X=1$ and $N$ is a maximal subgroup of $N X$; and we deduce from (6) that $E$ is a 2-uniserial subgroup of $N X$. It is a consequence of (7) that $G^{\prime} \subseteq N X$ and that therefore $N X$ is a normal subgroup of $G$; and consequently we deduce from our second hypothesis that $E$ is a normal subgroup of $N X$. Hence $E$ is normalized by $X$ so that $E$ is a maximal subgroup of $E X$. Application of (6) shows that $E X=N$, a contradiction. Hence it follows from (7) that

(8) $N$ is the set consisting of 1 and the elements of order $p$ in $G$.

Consider now a cyclic subgroup $S$ of $G$ whose order is at most $p^{2}$. If the order is less than $p^{2}$, then it follows from (8) and (7) that $E$ is centralized by $S$. If the order of $S$ is $p^{2}$, then we deduce $G^{\prime} \subset S$ from (3); and this implies $N \cap S=G^{\prime}$ by (7) and (8) so that $N S / N \simeq S / G^{\prime}$ is cyclic of order $p$. Then $E$ is by (6) a 2 -uniserial subgroup of $N S$; and $N S$ is a normal subgroup of $G$ by (2). Application of the second hypothesis shows that $E$ is a normal subgroup of $N S$. Since $S$ is a normal subgroup of $G$ by (3), the subgroups $E$ and $S$ normalize each other; and since

$$
E \circ S \subseteq E \cap S=E \cap N \cap S=E \cap G^{\prime}=1
$$

by (5), $E$ is centralized by $S$. Thus we have shown that $E$ is centralized by every element $x$ in $G$ whose order does not exceed $p^{2}$. Denote by $F$ the totality of elements $x$ in $G$ with $x^{p^{2}}=1$. An element $x$ belongs, by (8), to $F$ if, and only if, $x^{p}$ belongs to $N$; and this is equivalent to $(N x)^{p}=1$. Consequently $N \subseteq F \subseteq \mathbf{c} E$ and $F / N$ is the totality of elements in $G / N$ whose order is a divisor of $p$. But $G / N$ is by (7) an abelian $p$-group so that $F / N$ is a characteristic subgroup of $G / N$. This implies that $F$ is a normal subgroup of $G$; and thus we have shown: 
(9) The totality $F$ of elements of order dividing $p^{2}$ is a normal subgroup of $G$ with $N \subseteq F \subseteq \mathbf{c} E$.

Since $E$ is not a normal subgroup of $G$, it follows from (9) that (10) $F \cdot \mathrm{z} G \neq G$.

Let $A=G / N$, an abelian $p$-group by (7). Since $F / N$ is by (9) the totality of elements in $A$ of an order not exceeding $p$, it follows from (9), (10) that the maximum order $p^{m}$ of elements in $A$ exceeds $p$; in other words $1<m$. Denote by $N g$ an element of order $p^{m}$ in $A$. It is well known that $\{N g\}$ is a direct factor of $A$; see, for instance, Fuchs [p. 80, Corollary 24.6]. Hence $A=\{N g\} B$ where $B$ is some suitably selected complementary direct factor of $A$. It is a consequence of (1) and (7) that $A^{p}$ is part of the cyclic group $Z=N \cdot \mathrm{z} G / N$. Thus $Z$ contains in particular the cyclic group $\left\{N g^{p}\right\}$ of order $p^{m-1}$. Since $m-1$ is positive, it follows that $Z \cap\{N g\} \neq 1$; and this implies that $Z \cap B=1$, as $\{N g\} \cap B=1$, and as $\{N g\}$ and $Z$ contain the same elements of order $p$. But this implies $B^{p} \subseteq Z \cap B=1$. Hence $B$ is an elementary abelian subgroup of $A$; and it follows from (9) that $B \subseteq F / N$. Application of (10) shows that $Z B \subseteq Z(F / N) \subset A$. Now $A / B$ is isomorphic to the cyclic group $\{N g\}$ of order $p^{m}$; and thus it follows from $Z \cap B=1$ that the order of $Z$ is smaller than $p^{m}$. But $Z$ contains the cyclic group $\left\{N g^{p}\right\}$ of order $p^{m-1}$. Hence the order of $Z$ is exactly $p^{m-1}$; and this implies $Z=\left\{N g^{p}\right\}$. This is equivalent to $N\left\{g^{p}\right\}=N \cdot \mathrm{z} G$. Since $g^{p}$ belongs to $\mathrm{z} G$ by (1), we deduce $\mathrm{z} G=\left\{g^{p}\right\}(N \cap \mathrm{z} G)$ from Dedekind's modular law. But $N \cap \mathrm{z} G=G^{\prime}$ by (7) and the cyclicity of $\mathrm{z} G$; see (1). As the order of the cyclic group $\{N g\}$ is $p^{m}$ and $1<m$, it follows from (3) that $G^{\prime} \subset\{g\}$ and hence $G^{\prime} \subseteq\left\{g^{p}\right\}$ so that finally

$$
\mathrm{z} G=\left\{\mathrm{g}^{\not}\right\}(N \cap \mathrm{z} G)=\left\{\mathrm{g}^{\not}\right\} G^{\prime}=\left\{\mathrm{g}^{\not}\right\} .
$$

Next we note, using (8), that $N \cap\{g\}=N \cap \mathbf{z} G=G^{\prime}$. Since the order of $\{N g\}=N\{g\} / N \simeq\{g\} /(N \cap\{g\})=\{g\} / G^{\prime}$ is $p^{m}$, it follows that the order of $g$ is $p^{m+1}$ and that of $\mathrm{z} G=\left\{g^{p}\right\}$ is $p^{m}$.

We consider next a cyclic subgroup $S$ of order $p^{2}$. It follows from (3) and (1) that

$$
S^{p}=G^{\prime} \subseteq \mathbf{z} G=\left\{g^{p}\right\} ;
$$

and noting that $G^{\prime}=\left\{g^{p^{m}}\right\}$, it follows that there exists an element $s$ with $S=\{s\}$ and $s^{p}=g^{p^{m}}$. From (1) and $1<m$ we deduce that $g^{p^{m-1}}$ belongs to $\mathbf{z} G$. 
Hence

$$
\left(s^{-1} g^{p^{m-1}}\right)^{p}=s^{-p} g^{p^{m}}=1 ;
$$

and it follows from (8) that $s^{-1} g^{p^{m-1}}$ belongs to $N$ so that $s$ belongs to $N\{g\}$. If $f$ is an element of $F$, then either $f^{p}=1$ and $f$ belongs to $N$ by (8); or else $f$ has order $p^{2}$ and $f$ belongs to $N\{g\}$. Thus we have shown that $F \subseteq N\{g\}$. Noting that $B \subseteq F / N$, it follows that $B \subseteq\{N g\}$; but $B \cap\{N g\}=1$ so that $B=1$ and $G / N=A=\{N g\}$, showing $G=N\{g\}$. We recall that $G^{\prime}=N \cap\{g\}$ and that $N=E G^{\prime}$. Hence

$$
G=N\{g\}=E G^{\prime}\{g\}=E\{g\} \text { and } E \cap\{g\}=1 .
$$

Letting $C=\{g\}$, we have shown that

(11) $C$ is a cyclic normal subgroup of $G$ whose order is $p^{m+1}$ with $1<m$; and it is $G=C E$ and $1=C \cap E$; and $\mathrm{z} G=C^{p}$.

Since $E$ is abelian by (5), and since $E \cap \mathrm{z} G=1$ by (5), 1 is the only element in $E$ centralizing $C$. Hence $E$ is by (5) an elementary abelian $p$-group isomorphic to a group of automorphisms of the cyclic group $C$ of order $p^{m+1}$. Recalling the structure of the group of automorphisms of a cyclic $p$-group-see, for instance, Fuchs [p. 221, Theorem 58.1]-it follows that either $E$ is cyclic of order $p$ or else $p=2$ and $E$ is of order 4 . But in the latter case $E$ induces in $C$ all the automorphisms of order 2 ; in particular the automorphism mapping every element in $C$ upon its inverse is induced by an element $e$ in $E$. Then

$$
C^{2}=e^{\circ} C \subseteq G^{\prime}
$$

and this is impossible, since the order of $C^{2}$ is $2^{m}$ which is by (11) at least 4 whereas the order of $G^{\prime}$ is by (1) exactly 2. Thus we have shown:

(12) $E$ is cyclic of order $p$.

It is a consequence of (11) and (7) that $G / N$ is cyclic of order $p^{m}$. Hence $N$ is an $m$-uniserial subgroup of $G$. Combining this with (6) and (1.1) we see that

(13) $E$ is an $(m+1)$-uniserial subgroup of $G$.

The statements (1), (11), (12) and (13) contain all the claims made in our proposition.

Corollary 2.4. The group $G$ is a splitting extension of a cyclic group of order $p^{n}$ with $2<n$ by a cyclic group of order $p$ with $o\left(G^{\prime}\right)=p$ if, and only if, 
(a) $G$ is nilpotent,

(b) $G$ is not a dedekind group,

(c) every. proper epimorphic image of $G$ is a dedekind group and

(d) normal subgroups of $G$ normalize their 2-uniserial subgroups.

Proof. Assume first that $G$ is a splitting extension of a cyclic group $C$ of order $p^{n}$ with $2<n$ by a cyclic group of order $p$ with $o\left(G^{\prime}\right)=p$. Then the order of $G$ is $p^{n+1}$ and there exists a subgroup $E$ of order $p$ with $G=E C$ and $1=E \cap C$. Furthermore $C$ is a normal subgroup of $G$. If $G^{\prime}$ were not contained in $C$, then $G^{\prime} C$ would have order $p^{n+1}$ so that $G$ would be the direct product of its cyclic subgroups $C$ and $G^{\prime}$, implying the commutativity of $G$ and $G^{\prime}=1$, a contradiction. Hence $G^{\prime} \subseteq C$; and it follows from well known theorems [on $p$-groups] that the normal subgroup $G^{\prime}$ of order $p$ is contained in $\mathrm{z} G$; see, for instance, Hall [p. 49, Theorem 4.3.4].

$G$ would be abelian, if $\mathrm{z} G$ were not a proper part of $C$. But $G$ is not abelian, since $G^{\prime} \neq 1$. Hence $\mathrm{z} G \subseteq C^{p}$, as $C$ is cyclic. If $s$ is an element in $C$ and $x$ is an element in $G$, then

$$
\left(s^{p}\right)^{x}=\left(s^{x}\right)^{p}=[s(s \circ x)]^{p}=s^{p}\left(s^{\circ} x\right)^{p}=s^{p},
$$

since $G^{\prime}$ is of order $p$ and part of the center $\mathrm{z} G$. Hence $s^{p}$ belongs to $\mathrm{z} G$, proving $C^{p} \subseteq \mathbf{z} G$. Consequently $\mathbf{z} G=C^{p}$.

$G$ is nilpotent, since $G$ is a $p$-group; and it follows from the Structure Theorem that $G$ is not a dedekind group. If $N \neq 1$ is a normal subgroup of the $p$-group $G$, then $N \cap \mathrm{z} G \neq 1-$ see, for instance, Kurosh [II, p. 165]. But $G^{\prime}$ is the one and only one cyclic subgroup of order $p$ of the cyclic group $z G$ so that $G^{\prime}$ is the one and only one minimal normal subgroup of $G$. Hence every proper epimorphic image of $G$ is abelian and a fortiori a dedekind group.

From $G^{\prime} \subseteq \mathrm{z} G=C^{p}$ and $G=C E$ and $\Lambda=C \cap E$ we deduce that $G / \mathbf{z} G$ is the direct product of two cyclic groups of order $p$; and now it follows that $\mathbf{z} G=G^{p}$. Hence every maximal subgroup of $G$ is a cyclic extension of $\mathrm{z} G$ and as such abelian. Consequently every proper subgroup of $G$ is abelian and normalizes in particular its 2 -uniserial subgroups. If $S$ is a subgroup of index $p^{2}$ in $G$, then $C^{p^{2}} \subseteq S$. Since the order of $C$ is $p^{n}$ with $2<n$, it follows that $C^{p^{2}} \neq 1$. As $G^{\prime}$ is the uniquely determined subgroup of order $p$ of $C$, it follows that $G^{\prime} \subseteq C^{p^{2}} \subseteq S$, implying that every subgroup of index $p^{2}$ is a normal subgroup. In par- 
ticular therefore all 2-uniserial subgroups of $G$ are normal. This shows the necessity of the conditions (a)-(d).

The sufficiency of the conditions $(a)-(d)$ is an almost immediate consequence of Proposition 2.3.

Remark 2.5. It is worth noting that we have verified condition (d) in the following stronger form;

$\left(d^{\circ}\right)$ Every proper subgroup of $G$ is abelian; and every subgroup $S$ of $G$ whose index $[G: S]$ is a square of a prime contains $G^{\prime}$ and is consequently normal.

Thus $\left(d^{\circ}\right)$ is a consequence of $(a)-(d)$.

Remark 2.6. The groups characterized by Corollary 2.4 are determined by the following two properties:

(A) $G$ is a splitting extension of a cyclic group $C$ of order $p^{n}$ with $2<n$ by a cyclic group of order $p$; and

(B) $G^{\prime}$ is cyclic of order $p$.

In the course of the first part of the proof of Corollary 2.4 we have shown that (A) and (B) imply the following conditions:

( $\left.\mathrm{B}^{\prime}\right) \quad G^{p}=\mathbf{z} G$.

$\left(\mathrm{B}^{\prime \prime}\right)$ The group of automorphisms induced by $G$ in $C$ is of order $p$.

If a group meets requirement (A), then $C$ and $G / C$ are cyclic so that $G^{\prime}=G \circ C$. If furthermore $\left(\mathrm{B}^{\prime}\right)$ is satisfied, then $C^{p}=\mathrm{z} G$ and the group of automorphisms, inauced by $G$ in $C$, is cyclic of order $p$. This implies that $G \circ C$ is of order $p$, if $p$ is odd; and if $p=2$, it follows that either $G \circ C$ is of order 2 or else $\mathrm{z} G$ is of order 2 . But the latter case contradicts $\left(\mathrm{B}^{\prime}\right)$ and $2<n$; and thus we have shown that we may substitute $\left(B^{\prime}\right)$ for $(B)$. The preceding argument shows too that we may substitute $\left(\mathrm{B}^{\prime \prime}\right)$ for $(\mathrm{B})$ if, and only if, $p \neq 2$.

Proposition 2.7. If $p$ is a prime and $2<n$, then there exists one and essentially only one group $G$ with the following properties:

(A) $G$ is a splitting extension of a cyclic group of order $p^{n}$ by a cyclic group of order $p$.

(B) $G^{\prime}$ is cyclic of order $p$.

Proof. Denote by $C$ a cyclic group of $\operatorname{order} p^{n}$ with $\boldsymbol{p}$ a prime and $2<n$. Let $G$ be the group obtained by adjoining to $C$ an element $e$, subject to the 
following relations :

$$
e^{p}=1 \text { and } x^{e}=x^{1+p^{n-1}} \quad \text { for every } x \text { in } C .
$$

It is fairly obvious that this group $G$ meets the requirements (A) and (B) and that $G$ is up to isomorphisms the only group with these properties; see also the discussion of Remark 2.6.

Remark 2.8. Note that the above result would remain true for $n=2$, though not for $n=1$.

Remark 2.9. It is a consequence of Corollary 2.4, Remark 2.5 and Proposition 2.7 that there exist groups of class 2 which are not dedekind groups, though every subgroup normalizes its 2-uniserial subgroups. This invalidates a former claim of the author; see Baer [p. 429, Lemma 5.2]. We are indebted to Proff. Nyman and Sonneborn for bringing this mistake to our attention.

THEOREM 2 10. If the exponent of the group $G$ is $n$-th power free, and if every normal subgroup of $G$ normalizes its 2-uniserial subgroups, then the following properties of $G$ are equivalent:

(1) $G$ is a dedekind group.

(2) i-uniserial subgroups of $G$ [with $i<n]$ are normal.

(3) $G$ is nilpotent; and if the epimorphic image $H$ of $G$ is a splitting extension of a cyclic group of order $p^{k}$ with $2<k[<n]$ by a group of order $p$, then $H^{\prime}$ is not of order $p$.

Proof. If $n=1$, then $G=1$; and thus we may assume without loss in generality that $1<n$.

If (1) is true, then every subgroup of $G$ is normal so that (2) is a consequence of (1).

Assume next the validity of (2). From $1<\boldsymbol{n}$ we infer then that every maximal subgroup of $G$ is normal; and we infer the nilpotency of $G$ from Wielandt's Theorem; see, for instance, Hall [p. 155, Corollary 10.3.4]. Assume next by way of contradiction that the epimorphic image $H$ of $G$ is a splitting extension of a cyclic group of order $p^{k}$ with $2<k$ by a group of order $p$ and that $H^{\prime}$ is of order $p$. Application of Corollary 2.4 shows :

$H$ is nilpotent, not a dedekind group, though all its proper epimorphic images are dedekind groups, and normal subgroups of $H$ normalize their 2- 
uniserial subgroups.

Hence Proposition 2.3 is applicable. Consequently there exists a cyclic normal subgroup $C$ of $H$ and a subgroup $E$ of order $p$ of $H$ such that

$$
H=C E, 1=C \cap E ;
$$

$E$ is a $k$-uniserial subgroup of $H$.

Note that $p^{k}$ is the order of $C$, since $p^{k+1}$ is the order of $H$. If $E$ were a normal subgroup of $H$, then $H$ would be abelian as the direct product of its cyclic normal subgroups $C$ and $E$; and this is impossible, since $H^{\prime} \neq 1$. It is a consequence of (2) and Lemma 1.2, (b) that $i$-uniserial subgroups of $H$ with $i<n$ are normal. Hence $n \leq k$. Since the exponent of $G$ is $n$-th power free, so is the exponent of the epimorphic image $H$ of $G$. Hence $k<n$, a contradiction proving that (3) is a consequence of (2).

Assume finally by way of contradiction that (1) is not a consequence of (3). Then there exists an epimorphic image $J$ of $G$ with the following properties:

(a) $J$ is not a dedekind group.

(b) Every proper epimorphic image of $J$ is a dedekind group.

Since epimorphic images of nilpotent groups are nilpotent, we have furthermore :

(c) $J$ is nilpotent.

If $S$ is a 2-uniserial subgroup of the normal subgroup $N$ of $J$, and if $\sigma$ is an epimorphism of $G$ upon $J$, then the inverse image $N^{\sigma^{-1}}$ of $N$ is a normal subgroup of $G$; and it follows from Lemma 1.2, (b) that $S^{\sigma^{-1}}$ is a 2 -uniserial subgroup of $N^{\sigma^{-1}}$. By our general hypothesis $S^{\tau^{-1}}$ is a normal subgroup of $N^{\sigma^{-1}}$; and consequently $S$ is a normal subgroup of $N$. Thus we have shown:

(d) Normal subgroups of $J$ normalize their 2-uniserial subgroups.

An immediate application of Corollary 2.4 shows that the epimorphic image $J$ of $G$ is a splitting extension of a cyclic group of order $p^{j}$ with $2<j$ by a group of order $p$ with $J^{\prime}$ of order $p$; and this contradicts (3). Hence (1) is a consequence of (3).

Remark 2.11. Suppose that $G$ is of squarefree exponent and that 1-uniserial subgroups of $G$ are normal. This latter requirement is equivalent to the condition: every maximal subgroup of $G$ is normal; and this implies by Wielandt's 
Theorem that $G$ is nilpotent. $G$ satisfies conditions (2) and (3) of Theorem 2.10 and its exponent is 2 -nd power free. But such groups need certainly not be dedekind groups. This shows the indispensability of the hypothesis:

$(+)$ Normal subgroups of $G$ normalize their 2-uniserial subgroups.

It is a trivial remark that every dedekind group meets this requirement $(+)$.

Remark 2.12. If $p$ is an odd prime, then there exists a group $G$ of exponent $p$, generated by elements $a, b, c, d, e, f$, subject to the following conditions:

$\{a, b\}$ and $\{c, d\}$ and $\{e, f\}$ centralize each other;

$a^{\circ} b=c^{\circ} d=e^{\circ} f=g$ is an element of order $p$ in $\mathrm{z} G$.

Then $G$ is a nilpotent group of square free exponent; and it is a consequsnce of the Structure Theorem that $G$ is not a dedekind group. From $G^{p}=1$ we infer the validity of (3); and the validity of (2) is a consequence of the fact that 1-uniserial subgroups of $G$ are normal as maximal subgroups of a $p$-group. Every 2-uniserial subgroup of $G$ has index $p^{2}$ in $G$; and such a subgroup certainly contains $g$ and hence $G^{\prime}$ so that it is a normal subgroup. Thus condition $(+)$ of Remark 2.11 is satisfied in the following weak form:

$\left(+{ }^{\circ}\right) \quad 2$-uniserial subgroups of $G$ are normal.

This example shows the impossibility of substituting in Theorem 2.10 for condition $(+)$ the weaker requirement $\left(+{ }^{\circ}\right)$.

Remark 2.13. That conditions (2) and (3) cannot be essentially weakened or omitted altogether is shown in the discussion of Remark 2.9.

Corollary 2.14. The group $G$ is a dedekind group if, and only if, (a) every uniserial subgroup of $G$ is a normal subgroup of $G$ and (b) normal subgroups of $G$ normalize their 2-uniserial subgroups.

This is an almost immediate consequence of Theorem 2.10.

Corollary 2.15. The group $G$ is a dedekind group of $n$-th power free exponent if, and only if,

(a) $i$-uniserial subgroups of $G$ with $i<n$ are normal subgroups of $G$

(b) normal subgroups of $G /\left(G \circ G^{\prime}\right)$ normalize their 2-uniserial subgroups and

(c) $G /\left(G \circ G^{\prime}\right)$ is of $n$-th power free exponent.

This one derives without too much difficulty from Theorem 2.10, Corollary 
2.2 and Lemma 1.2.

Observation 2.16. The property of being a dedekind group is inherited by subgroups and epimorphic images. Our criteria involve conditions asserting that certain normal subgroups normalize some of their uniserial subgroups; and such a condition is prima facie not inherited by subgroups, though as a consequence of Lemma 1.2 it is inherited by epimorphic images.

\section{Groups whose proper subgroups are dedekind groups}

The basis of our discussion is the following fairly direct application of the Theorems of Iwasawa-Rédei-Schmidt.

Proposition 3.1. The following properties of the non-nilpotent group $G$ are equivalent :

( $i$ ) Every proper subgroup of $G$ is a dedekind group.

(ii) If a proper subgroup of $G$ is non-abelian, then it is the direct product of a quaternion group and a cyclic 3-group.

(iii) $G$ is an extension of a p-group $P$ by a cyclic $q$-group with $p \neq q$, meeting the following requirements:

(a) $[G: P \cdot \mathbf{c} P]=q$.

(b) Either $P$ is a quaternion group and $q=3$; or else $P$ is an elementary abelian p-group and a minimal normal subgroup of $G$.

Proof. Assume first that every proper subgroup of $G$ is a dedekind group. Since dedekind groups are, by the Structure Theorem of $\S 2$, nilpotent, $G$ is a non-nilpotent group all of whose proper subgroups are nilpotent. Application of the Theorem of Iwasawa-Schmidt shows the existence of two different primes $p$ and $q$ such that

(1) $G^{\prime}$ is a $p$-group and $G / G^{\prime}$ is a cyclic $q$-group.

See Rédei [p. 304, Satz 1].

Since $G$ is not nilpotent, there exist $q$-elements which do not centralize $G^{\prime}$ and this implies $G^{\prime} \cdot \mathbf{c} G^{\prime} \subset G$. Since $G / G^{\prime}$ is a cyclic $q$-group, there exists one and only one subgroup $V$ of $G$ with $G^{\prime} \cdot \mathbf{c} G^{\prime} \subseteq V$ and $[G: V]=q$. As a proper subgroup of $G$ is a dedekind group and hence nilpotent, $V$ is the direct of the $p$-Sylow subgroup $G^{\prime}$ of $G$ and $V$ and of a $q$-group; and this implies clearly $V=G^{\prime} \cdot \mathbf{c} G^{\prime}$. Hence $\left[G: G^{\prime} \cdot \mathbf{c} G^{\prime}\right]=q$. 
If firstly $G^{\prime}$ is abelian, then every proper subgroup of $G$ is abelian, since proper subgroups of $G$ are direct products of subgroups of $G^{\prime}$ and of cyclic $q$ groups, and we deduce from a result of Rédei [p. 305, Satz 2] that $G^{\prime}$ is an elementary abelian $p$-group and a minimal normal subgroup of $G$.

If secondly $G^{\prime}$ is not abelian, then we recall that $G^{\prime}$, as a proper subgroup of $G$, is a dedekind group. It is a consequence of the Structure Theorem of $\S 2$ that $G^{\prime}$ is the direct product of a quaternion group and an elementary abelian 2-group, since $G^{\prime}$ is primary. It follows in particular that

$\mathrm{z} G^{\prime}$ is the totality of elements $x$ in $G^{\prime}$ with $x^{2}=1$ and as such an elementary abelian 2-group, and that

$G^{\prime} / \mathbf{z} G^{\prime}$ is the direct product of two cyclic groups of order 2 .

If we adjoin to $\mathrm{z} G^{\prime}$ any $q$-element of $G$, then we obtain a proper subgroup of $G$ which is nilpotent. Hence $\mathbf{z} G^{\prime}$ is centralized by all the $q$-elements in $G$; and we deduce

$$
\mathbf{z} G^{\prime} \subseteq \mathbf{z} G
$$

from (1).

From $\left[G: G^{\prime} \mathbf{c} G^{\prime}\right]=q$ we deduce that an automorphism $\sigma$ of order $q$ is induced in $G^{\prime}$ by some element in $G$. From $\mathbf{z} G^{\prime} \subseteq \mathbf{z} G$ we deduce that every element in $\mathrm{z} G^{\prime}$ is a fixed element of $\sigma$. If $\sigma$ would induce in $G^{\prime} / \mathbf{z} G^{\prime}$ the identity automorphism, then $\sigma^{2}=1$ would follow from $\left(\mathrm{z} G^{\prime}\right)^{2}=1$; and this would imply $q=2=p$, an impossibility. Consequently $\sigma$ induces in $G^{\prime} / \mathbf{z} G^{\prime}$ an automorphism of order $q \neq 2$; and since $G^{\prime} / \mathbf{z} G^{\prime}$ is an elementary abelian group of order 4 , it follows that $q=3$.

Since $G^{\prime}$ contains a quaternion group, it contains an element $a$ of order 4 . If we let $a^{\sigma}=b$, then

$$
b^{\sigma} \equiv a b \text { modulo } \mathbf{z} G^{\prime}
$$

since the automorphism, induced by $\sigma$ in the elementary abelian group $G^{\prime} / \mathbf{z} G^{\prime}$ of order 4, has order 3. Hence there exists an element $c$ in $\mathrm{z} G^{\prime}$ with $b^{s}=a b c$. Let $a^{*}=a c$. Then $b^{*}=a^{* \sigma}=b c$, since every element in $\mathrm{z}^{\prime}$ is a fixed element of $\sigma$; and

$$
b^{* \sigma}=(b c)^{\sigma}=a b c^{2}=(a c)(b c)=a^{*} b^{*} .
$$

It follows that $Q=\left\{a^{*}, b^{*}\right\}$ is a quaternion subgroup of $G$, which is left invariant by $\sigma$ and in which $\sigma$ induces an automorphism of order 3 . There exists a 3 - 
element $s$ in $G$ which induces the automorphism $\sigma$ of order 3 in $G^{\prime}$. The subgroup $\{Q, s\}=Q\{s\}$ of $G$ is not nilpotent, hence not a dedekind group and hence not a proper subgroup of $G$ [by (i)]. Thus $G=Q\{s\}$ with $Q$ a normal 2-subgroup of $G$ and $G / Q$ a cyclic 3-group; and this implies $Q=G^{\prime}$.

If we let $P=G^{\prime}$, then our considerations show that we have deduced (iii) from (i).

It is fairly immediate that (ii) is a consequence of (iii); and it is a consequence of $\S 2$, Structure Theorem that (ii) implies (i).

Corollary 3.2. If the group $G$ is not nilpotent, though every proper subgroup of $G$ is a dedekind group, then the following properties of $G$ are equivalent:

( $i$ ) Maximal subgroups of maximal subgroups of $G$ are normal subgroups of G.

(ii) 2-uniserial subgroups of $G$ are normal.

(iii) $G^{\prime}$ is cyclic.

(iv) Proper subgroups of $G$ are cyclic.

Proof. Application of Proposition 3.1 shows that

(1) $G^{\prime}$ is a $p$-group and $G / G^{\prime}$ is a cyclic $q$-group with $p \neq q$,

(2) $\left[G: G^{\prime} \cdot \mathbf{c} G^{\prime}\right]=q$,

(3) either $G^{\prime}$ is a quaternion group and $q=3$ or else $G^{\prime}$ is an elementary abelian $p$-group and a minimal normal subgroup of $G$.

Naturally (ii) is a special case of (i) and hence a consequence of (i). Assume next the validity of (ii). If $G^{\prime}$ were a quaternion group and $q=3$, then we deduce from (1) the existence of a cyclic 3 -subgroup $S$ of $G$ with $G=G^{\prime} S$. Then $S$ centralizes $\mathrm{z} G^{\prime}$ and induces in $G^{\prime} / \mathbf{z} G^{\prime}$ a group of automorphisms of order 3 , as follows from (2). Since $G^{\prime}$ is a quaternion group and $\mathrm{z} G^{\prime}$ is the one and only one minimal subgroup of $G^{\prime}$, the only subgroup of $G$ which is properly situated between $S$ and $G$ is $S \cdot z G^{\prime}$. Hence $S$ is a 2 -uniserial subgroup of $G$ which is by (ii) a normal subgroup of $G$. Since $S$ and $G^{\prime}$ are of relatively prime order, these two normal subgroups of $G$ centralize each other so that $G=G^{\prime} \mathbf{c} G^{\prime}$, contradicting (2). Thus it follows from (3) that $G^{\prime}$ is an elementary abelian $p$-group and a minimal normal subgroup of $G$. Naturally $G^{\prime}$ possesses a subgroup $J$ with $\left[G^{\prime}: J\right]=p$. Again we deduce from (1) the existence of a cyclic $q$-subgroup $S$ of $G$ with $G=G^{\prime} S$; and it follows from (2) and the com- 
mutativity of $G^{\prime}$ that

$$
\mathbf{c} G^{\prime}=G^{\prime} S^{q}
$$

Assume by way of contradiction that $J \neq 1$. Then it follows from the minimality and commutativity of $G^{\prime}$ that $G^{\prime}=\left\{J^{S}\right\}$ so that $G=\{J, S\}$. Hence the only subgroup of $G$ which is properly contained between $J S^{q}$ and $G$ is $G^{\prime} S^{q}=\mathbf{c} G^{\prime}$. Consequently $J S^{q}$ is a 2 -uniserial subgroup of $G$; and it follows from (ii) that $J S^{q}$ is a normal subgroup of $G$. But $J$ is the $p$-component of $J S^{q}$; and as such $J$ is a characteristic subgroup of the normal subgroup $J S^{q}$ of $G$. Hence $J$ is a normal subgroup of $G$ with $1 \subset J \subset G^{\prime}$, contradicting the minimality of $G^{\prime}$. This contradiction shows that $J=1$ so that $G^{\prime}$ is of order $p$; and we have deduced (iii) from (ii).

If $G^{\prime}$ is cyclic, then the order of $G^{\prime}$ is a prime $p$ by (3) and we deduce from (1), (2) the existence of a cyclic $q$-subgroup $S$ of $G$ with

$$
G=G^{\prime} S \text { and } \mathbf{c} G^{\prime}=G^{\prime} S^{q} .
$$

Now one sees without difficulty that a maximal subgroup of $G$ is either a complement of $G^{\prime}$ or else contains $G^{\prime}$. In the first case this maximal subgroup is a cyclic $q$-group and in the second case it is the uniquely determined subgroup c $G^{\prime}$ of index $q$ in $G$ and in both cases it is cyclic. Thus (iv) is a consequence of (iii).

In the presence of (iv) we may apply Proposition 3.1 to see that $G$ is an extension of a minimal normal subgroup $P$ of order $p$ by a cyclic $q$-group [with $p \neq q]$. Furthermore $[G: \mathbf{c} P]=q$ so that the characteristic subgroup $\mathbf{c} P$ of $G$ is cyclic. Now one verifies that the maximal subgroups of maximal subgroups are $(\mathbf{c} P)^{p}$ and $(\mathbf{c} P)^{q}$ if $\neq \mathbf{c} P$; and these are characteristic so that (i) is a consequence of (iv).

Theorem 3.3. The following properties of the group $G$ are equivalent:

(i) $G$ is a dedekind group.

(ii) $\left\{\begin{array}{l}\text { (a) Subgroups of } G \text { normalize their 2-uniserial subgroups. } \\ \text { (b) If the factor } F \text { of } G \text { is a splitting extension of a cyclic group by a } \\ \text { cyclic group, then } F^{\prime} \text { is not of order a prime. }\end{array}\right.$ (iii) $\left\{\begin{array}{l}\text { (a) 2-uniserial subgroups of } G \text { are normal. } \\ \text { (b) A factor of } G \text { is a dedekind group, if it is nilpotent or of squarefree } \\ \text { exponent. }\end{array}\right.$ 
Here as always we term factor of the group $X$ every epimorphic image of a subgroup of $X$.

Proof. If $G$ is a dedekind group, then all the subgroups of $G$ are normal, implying the validity of (ii.a). It follows furthermore from $\S 2$, Structure Theorem that non-abelian dedekind groups contain quaternion groups and are consequently not splitting extensions of cyclic groups by cyclic groups. Since factors of dedekind groups are likewise dedekind groups, $G$ meets requirement (ii. b). Thus (ii) is a consequence of (i).

Assume next the validity of (ii). It is a consequence of Lemma 1.2, (b) that every factor of $G$ normalizes its 2 -uniserial subgroups. Consider now some factor $F$ of $G$ which is nilpotent or of sqarefree exponent. If $F$ were not a dedekind group, then $F$ would possess a factor $E$ of minimal order which is not a dedekind group. We note that $E$ as a factor of $F$ is nilpotent or of squarefree exponent, that subgroups of $E$ normalize their 2-uniserial subgroups and that $o\left(S^{\prime}\right)$ is not a prime in case the factor $S$ of $E$ is a splitting extension of a cyclic group by a cyclic group.

Assume that $E$ is nilpotent. Since $E$ is not a dedekind group, though every proper epimorphic image is a dedekind group, we may apply Corollary 2.4. Hence $E$ is a $p$-group, a splitting extension of a cyclic group by a cyclic group with $E^{\prime}$ of order $p$; and this is impossible. Thus $E$ is not nilpotent.

Since $E$ is not nilpotent, it is of squarefree exponent. Since proper subgroups of $E$ are dedekind groups, we may apply Corollary 3.2. Hence every proper subgroup of $E$ is cyclic and application of Proposition 3.1 shows now that $E^{\prime}$ is cyclic of order a prime $p$ and $E / E^{\prime}$ is cyclic of order a prime $q \neq p$, since $E$ is of squarefree exponent. Thus $E$ is a splitting extension of a cyclic group by a cyclic group with $E^{\prime}$ of order a prime; and this contradicts (ii.b), since $E$ is a factor of $G$. Hence (iii) is a consequence of (ii).

If (i) were not a consequence of (iii), then there would exist groups, meeting requirement (iii), without being dedekind groups; and among these groups there is one $G$ of minimal order. Clearly every proper factor of $G$ is a dedekind group; and $G$ itself is neither nilpotent nor of squarefree exponent. Thus we may apply Proposition 3.1 and Corollary 3.2. It follows that $G^{\prime}$ is cyclic of order $p$, that $G / G^{\prime}$ is a cyclic group of order $q^{n}$ with $q \neq p$ and that $\mathrm{z} G$ is of order $q^{n-1}$. Since $G$ is not of squarefree exponent, we have $1<n$. 
Hence $G / \mathrm{z} G$ is a proper factor of $G$ and as such it is a dedekind group. Since $G / \mathrm{z} G$ is of order $p q$ with $p \neq q$, it follows from $\S 2$, Structure Theorem that $G / \mathbf{z} G$ is abelian. Since $1=G^{\prime} \cap \mathbf{z} G$, this implies $G^{\prime}=1$ in contradiction to our choice of $G$ as a non-dedekindian group. Hence (i) is a consequence of (iii).

Remark 3.4. The existence of nilpotent groups, meeting requirement (ii. a) without being dedekind groups, has been shown in $\S 2$; see Remark 2.9. The non-abelian groups of order $p q$ with $p$ a prime and $q$ a prime divisor of $p-1$ provide examples of non-nilpotent groups, satisfying condition (2.a). Thus we see that codition (2. b) is indispensable. The indispensability of (2.a) is an immediate consequence of $\S 2$, Structure Theorem; and likewise one sees that it is impossible to omit in (iii) the nilpotent factors or the factors of squarefree exponent or the second half of condition (iii).

\section{Literature}

[1] Reinhold Baer, Nilpotent groups and their generalisations, Trans. Amer. Math. Soc. 47, 393-434 (1940).

[2] L. Fuchs, Abelian groups, Budapest (1958).

[3] Marshall Hall, Jr., The Theory of Groups, New York (1959).

[4] A. G. Kurosh, The Theory of Groups; 2nd english edition, New York (1960).

[5] L. Rédei, Die endlichen einstufig nicht nilpotenten Gruppen, Publications Math. Debrecen 4, 303-324 (1956).

\section{Frankfurt University}

